EDITORIAL

\title{
Editor's Introduction to This Issue
}

\author{
Yeun-Jun Chung* \\ IRCGP, The Catholic University of Korea, College of Medicine, Seoul 137-701, Korea
}

Since the advent of next-generation sequencing, we have realized that a substantial amount of the human genome, not just the $2 \%$ to $5 \%$ of the human genome that is occupied by coding genes, is transcribed, which means that there is a substantial amount of non-protein-coding RNAs in the human genome. Non-coding RNAs are RNA transcripts that are not translated into proteins; some of them play a role in the regulation of gene expression and will be eventually implicated in on diverse diseases, including cancer. In this issue, Dr. Yong Sun Lee (University of Texas) reviewed the implications of a novel type of non-coding RNA, nc886, which was recently discovered by his group. nc886 is known to have putative tumor suppressor and tumor-sensing functions. In this review, the characteristics and roles of this
ncRNA in tumorigenesis are introduced. Dr. Namshin Kim's group (KOBIC) reported the effect of exome sequencing depth on the discovery of sequence variants for clinical use. They suggested the optimal sequencing depth for defining most diverse function-related variants, which would be useful for next-generation sequencing based biomedical research and clinical translation. Dr. Jung Kyoon Choi's group (KAIST) investigated how transcription and epigenetic processes are genetically regulated and associated with each other. For this purpose, they examined the changes in chromatin accessibility and gene expression in response to genetic variation by means of quantitative trait loci mapping. In addition, there are three articles on in silico modeling. 